VOL. 75 (2007) [179-191]

\title{
TRANSFORMATIONS FOR COMPLEX DISCRETE LINEAR HAMILTONIAN AND SYMPLECTIC SYSTEMS
}

\author{
Yi Wang, Yuming Shi and Guojing Ren
}

This paper is concerned with transformations for complex discrete linear Hamiltonian systems and complex discrete linear symplectic systems. A general complex discrete trigonometric system is studied and a criterion for it is established. Based on these results, the Prüfer and trigonometric transformations for complex discrete linear Hamiltonian systems and complex discrete linear symplectic systems are formulated. The relative existing results in real cases are extended.

\section{INTRODUCTION}

The field of difference equations is a mathematically rich and versatile area that is used for modelling discrete processes. The interest in studying difference equations has been created and is sustained by the following two main factors: one is due to the theoretical significance and diverse modelling applications to almost all areas of science, engineering, and technology where discrete phenomena abound; and the other one is that with the wide applications of digital computer, differential equations are solved by employing their approximative difference-equation formulations. Thus the need for, and interest in scientific advancement in the area is naturally motivated [12]. For more motivation, we refer the reader to $[\mathbf{1 0}, \mathbf{1 6}]$.

In this paper, we consider the following complex discrete linear Hamiltonian system:

$$
\begin{aligned}
& \Delta x(t)=A(t) x(t+1)+B(t) u(t), \\
& \Delta u(t)=K(t) x(t+1)-A^{*}(t) u(t), \quad t \in \mathcal{I},
\end{aligned}
$$

where the interval $\mathcal{I}:=[a, \infty)$ is the integer set $\{t\}_{t=a}^{\infty} ; \Delta$ is the forward difference operator: $\Delta x(t)=x(t+1)-x(t) ; A(t), B(t)$, and $K(t)$ are $n \times n$ complex matrices; $A^{*}(t)$ denotes the complex conjugate transpose of $A(t)$; and $B(t)$ and $K(t)$ are Hermitian. We also consider the following complex discrete symplectic system:

$$
z(t+1)=\Phi(t) z(t), \quad t \in \mathcal{I}
$$

Received 19th June, 2006

This research was supported by the NNSF of China (Grant 10471077) and Shandong Research Funds for Young Scientists (Grant 03BS094).

The authors are indebted to the referees for their constructive and detailed suggestions.

Copyright Clearance Centre, Inc. Serial-fee code: 0004-9727/07 \$A2.00+0.00. 
where $\Phi(t)$ is a $2 n \times 2 n$ complex symplectic matrix for all $t \in \mathcal{I}$, that is, $\Phi^{*}(t) J \Phi(t)=J$, while

$$
J=\left(\begin{array}{cc}
0 & I_{n} \\
-I_{n} & 0
\end{array}\right)
$$

is the canonical symplectic matrix.

Throughout the whole paper, we always assume that $I_{n}-A(t)$ is nonsingular in $\mathcal{I}$, where $I_{n}$ is the $n \times n$ unit matrix. Under this assumption, system (1.1) can be written as system (1.2). Their relationships will be discussed in detail in Section 2.

It is well known that both the Prüfer transformation and the trigonometric transformation are very useful in the study of the Sturm-Liouville theory and oscillation problems for second-order ordinary differential equations. Later, Barrett [4], Reid [13], and Zheng [17, Chapter 2, Section 3] formulated the Prüfer transformation for the complex linear continuous Hamiltonian system

$$
\begin{aligned}
& x^{\prime}(t)=A(t) x(t)+B(t) u(t), \\
& u^{\prime}(t)=K(t) x(t)-A^{*}(t) u(t)
\end{aligned}
$$

by using the so-called complex trigonometric system

$$
s^{\prime}(t)=Q(t) c(t), \quad c^{\prime}(t)=-Q(t) s(t),
$$

where $A(t), B(t), K(t)$, and $Q(t)$ are $n \times n$ continuous matrix-valued functions in the continuous interval $[a,+\infty)$, and $B(t), K(t)$, and $Q(t)$ are Hermitian. Doššlý gave a trigonometric transformation, which can turn a real linear continuous Hamiltonian system into a real trigonometric system [9]. These transformations are also useful in the study of oscillation problems for linear continuous Hamiltonian systems.

For discrete cases, relative problems were investigated and some good results have been obtained. The key to establishing the Prüfer and trigonometric transformations is discrete trigonometric systems. In 1997, Anderson introduced a special discrete trigonometric system

$$
\begin{aligned}
& X(t+1)=\cos Q(t) X(t)+\sin Q(t) U(t), \\
& U(t+1)=-\sin Q(t) X(t)+\cos Q(t) U(t), \quad t \in \mathcal{I},
\end{aligned}
$$

where $Q(t)$ is a $n \times n$ Hermitian matrix-valued function in the interval $\mathcal{I}$, and $\sin Q(t)$ and $\cos Q(t)$ are defined by the sine and cosine Maclaurin series [3]. He gave a definition of discrete matrix-valued sinusoidal functions by solutions of certain initial value problems of system (1.3), discussed their properties, and established an oscillation criterion for system (1.3). Recently, Bohner and Došlý studied the general real discrete trigonometric system

$$
\begin{aligned}
& x(t+1)=P(t) x(t)+Q(t) u(t) \\
& u(t+1)=-Q(t) x(t)+P(t) u(t),
\end{aligned}
$$


where $P(t)$ and $Q(t)$ are $n \times n$ real matrix-valued functions and satisfy

$$
P^{T}(t) P(t)+Q^{T}(t) Q(t)=I_{n}, P^{T}(t) Q(t)=Q^{T}(t) P(t)
$$

(see $[6,7,8]$ ). In $[6,7]$, they gave a discrete trigonometric transformation for a real discrete symplectic system and applied the transformation to establish an oscillation criterion for the real symplectic system. In [8], they formulated the discrete Prüfer transformation for the real scalar self-adjoint second-order difference equation

$$
\Delta\left(r_{k} \Delta x_{k}\right)+p_{k} x_{k+1}=0
$$

and employed the transformation to obtain an oscillation criterion for equation (1.4). In $[7,8]$, they also introduced the discrete Prüfer transformations for a real discrete symplectic system and for the real symplectic dynamic system

$$
z^{\Delta}(t)=S(t) z(t)
$$

on the time scale $\mathbf{T}$, respectively, where $z^{\Delta}(t)$ is the delta-derivative of $z$ at $t \in \mathbf{T}$.

However, there appear to be few studies concerning transformations for complex discrete linear Hamiltonian systems and complex discrete linear symplectic systems. It is noted that in order to formulate the transformations in $[\mathbf{6}, \mathbf{7}, \mathbf{8}]$, Bohner and Došlý constructed a unitary matrix whose real part or imaginary part is composed of a coefficient matrix and one component of a real conjoined basis or normalised conjoined bases, which are complex in complex cases. Hence, the methods employed in real cases in $[\mathbf{6}, \mathbf{7}, \mathbf{8}]$ can not be directly applied in complex cases. The present paper will formulate Prüfer and trigonometric transformations for complex discrete linear Hamiltonian systems and complex discrete linear symplectic systems by applying a criterion of complex discrete trigonometric systems.

The rest of this paper is organised as follows. Section 2 collects some basic concepts and some useful lemmas. A complex discrete trigonometric system is studied and a criterion of it is established. In Section 3, Prüfer and trigonometric transformations for complex discrete linear Hamiltonian systems and complex discrete linear symplectic systems are investigated.

\section{Preliminaries}

We first discuss the relationships between the Hamiltonian system (1.1) and the symplectic system (1.2). If $I_{n}-A(t)$ is invertible in $\mathcal{I}$, then system (1.1) can be written as a special symplectic system (1.2) with

$$
\Phi(t)=\left(\begin{array}{cc}
E & E B \\
K E & E^{*-1}+K E B
\end{array}\right)(t),
$$


$E(t):=(I-A(t))^{-1}$ (see [1, Example 2.4], [2, Theorem 3.19], and [14, Theorem 2.1]). However, the symplectic system (1.2) with

$$
\Phi(t)=\left(\begin{array}{ll}
A_{11} & A_{12} \\
A_{21} & A_{22}
\end{array}\right)(t)
$$

can not be turned into a Hamiltonian system (1.1) in general unless that $A_{11}(t)$ is invertible for all $t \in \mathcal{I}$, where $A_{i j}(t), 1 \leqslant i, j \leqslant 2$, are $n \times n$ matrices. So we shall focus our attention on the study of system (1.2) and the relative results for system (1.1) can be directly derived from the above relationship in the present paper.

Next, we investigate properties of solutions of system (1.2). The following result can be easily derived from the symplectic property of $\Phi(t)$.

LEMMA 2.1. Assume that $\left(X_{1}^{T}, \dot{U}_{1}^{T}\right)^{T}(t)$ and $\left(X_{2}^{T}, U_{2}^{T}\right)^{T}(t)$ are two $2 n \times n$ matrixvalued solutions of system (1.2). Then there exists a $n \times n$ constant matrix $C$ such that

$$
\left(X_{1}^{*}, U_{1}^{*}\right)(t) J\left(\begin{array}{l}
X_{2} \\
U_{2}
\end{array}\right)(t)=C, \quad \forall t \in \mathcal{I} .
$$

DEFINITION 2.1:

(1) Let $\left(X^{T}, U^{T}\right)^{T}(t)$ be a $2 n \times n$ matrix-valued solution of system (1.2). If $X^{*}(t) U(t)$ is Hermitian and $\operatorname{rank}(X, U)(t)=n$ for all $t \in \mathcal{I}$, then $\left(X^{T}, U^{T}\right)^{T}(t)$ is said to be a conjoined basis of system (1.2).

(2) Two conjoined bases $\left(X_{i}^{T}, U_{i}^{T}\right)^{T}(t)$ of system (1.2), $i=1,2$, are said to be normalised conjoined bases of system (1.2) if

$$
\left(X_{1}^{*}, U_{1}^{*}\right)(t) J\left(\begin{array}{l}
X_{2} \\
U_{2}
\end{array}\right)(t) \equiv I_{n}, \forall t \in \mathcal{I} .
$$

(3) If normalised conjoined bases $\left(X_{i}^{T}, U_{i}^{T}\right)^{T}(t)$ of system (1.2), $i=1,2$, satisfy

$$
X_{1}(a)=U_{2}(a)=0, U_{1}(a)=-X_{2}(a)=I_{n},
$$

then they are said to be special normalised conjoined bases of system (1.2) at $t=a$.

REMARK 2.1. (i) Similar concepts in (1) and (2) of Definition 2.1 can be found in [2, pp. 114 and 120], in which they are called a prepared basis and a normal pair of solutions, respectively. The concepts in Definition 2.1 are similar to those defined for real discrete linear Hamiltonian systems in [15, Section 1.4] and for real continuous linear Hamiltonian systems in [11, Chapter 1, Section 1].

(ii) With a similar argument to that in [15, Lemma 1.4.2] for real discrete linear Hamiltonian systems, it can be proved that there exist a conjoined basis of (1.2) and 
special normalised conjoined bases of system (1.2) at any given $t_{0} \in \mathcal{I}$ by Lemma 2.1 and using the symplectic property of $\Phi$.

Lemma 2.2. Assume that $\left(X_{i}^{T}, U_{i}^{T}\right)^{T}(t), i=1,2$, are special normalised conjoined bases of system (1.2) at $t=a$. Then the matrix

$$
D(t)=\left(\begin{array}{ll}
X_{1} & X_{2} \\
U_{1} & U_{2}
\end{array}\right)(t)
$$

is symplectic in $\mathcal{I}$.

Proof: By Lemma 2.1, for any $t \in \mathcal{I}$ we have

$$
D^{*}(t) J D(t)=D^{*}(a) J D(a)=\left(\begin{array}{cc}
0 & -I_{n} \\
I_{n} & 0
\end{array}\right)^{*} J\left(\begin{array}{cc}
0 & -I_{n} \\
I_{n} & 0
\end{array}\right)=J .
$$

This completes the proof.

Lemma 2.3. Assume that $S$ is a $2 n \times 2 n$ complex matrix. Then $S$ is symplectic if and only if $S^{*}$ is symplectic.

ProOF. The proof is elementary and so we omit its details.

The following result is a direct consequence of Lemmas 2.2 and 2.3.

LEMma 2.4. If $\left(X_{i}^{T}, U_{i}^{T}\right)^{T}(t), i=1,2$, are special normalised conjoined bases of system (1.2) at $t=a$, then $\left(X_{1} X_{2}^{*}\right)(t)=\left(X_{2} X_{1}^{*}\right)(t),\left(U_{1} U_{2}^{*}\right)(t)=\left(U_{2} U_{1}^{*}\right)(t)$, and $\left(X_{1}^{*} U_{2}-U_{1}^{*} X_{2}\right)(t)=I_{n}$ for all $t \in \mathcal{I}$.

The next lemma is simple, but useful in the present paper.

Lemma 2.5. Let $P$ and $Q$ be two $n \times n$ complex matrices. Then

$$
P^{*} P+Q^{*} Q=I, \quad P^{*} Q-Q^{*} P=0
$$

if and only if

$$
P P^{*}+Q Q^{*}=I, P Q^{*}-Q P^{*}=0
$$

Proof: Denote

$$
G:=\left(\begin{array}{cc}
P & Q \\
-Q & P
\end{array}\right)
$$

It can be easily proved that (2.2) and (2.3) are equivalent to $G^{*} J G=J$ and $G J G^{*}=J$, respectively. So this lemma follows from Lemma 2.3. This completes the proof.

We now introduce a complex discrete trigonometric system and discuss properties of its some special solution.

DEFINITION 2.2: The system

$$
\begin{aligned}
& x(t+1)=P(t) x(t)+Q(t) u(t), \\
& u(t+1)=-Q(t) x(t)+P(t) u(t),
\end{aligned}
$$


is called a complex discrete trigonometric system if $P(t)$ and $Q(t)$ are $n \times n$ complex matrices and satisfy $(2.2)$ for all $t \in \mathcal{I}$.

Obviously, it follows from the proof of Lemma 2.5 that system (2.4) is a special symplectic system. By $\left(S^{T}, C^{T}\right)^{T}(t)$ denote the $2 n \times n$ matrix-valued solution of system (2.4) with the initial condition

$$
X(a)=0, U(a)=I_{n}
$$

Using the similar methods to those employed in the proofs of $[3$, Theorem 5 and Corollary 8], one can show the following Propositions 2.1 and 2.2 .

Proposition 2.1. For each $t \in \mathcal{I}, S(t)$ and $C(t)$ satisfy

$$
\begin{aligned}
& \left(S^{*} S+C^{*} C\right)(t)=I_{n}, \quad\left(S^{*} C\right)(t)=\left(C^{*} S\right)(t), \\
& \left(S S^{*}+C C^{*}\right)(t)=I_{n}, \quad\left(S C^{*}\right)(t)=\left(C S^{*}\right)(t), \quad\|S(t)\|_{2}^{2}+\|C(t)\|_{2}^{2}=n,
\end{aligned}
$$

where $\|A\|=\left(\sum_{i, j=1}^{n}\left|a_{i j}\right|^{2}\right)^{1 / 2}$ for the $n \times n$ matrix $A=\left(a_{i j}\right)_{n \times n}$.

Proposition 2.2. For each $t \in \mathcal{I}, S(t)$ and $C(t)$ satisfy

$$
\begin{aligned}
& S(t+1) S^{*}(t)+C(t+1) C^{*}(t)=P(t), \\
& S(t+1) C^{*}(t)-C(t+1) S^{*}(t)=Q(t),
\end{aligned}
$$

where $P(t)$ and $Q(t)$ are the coefficient matrices in system (2.4).

At the end of this section, we give a criteria of complex trigonometric systems.

THEOREM 2.1. Let $P(t)$ and $Q(t)$ be $n \times n$ complex matrices. If system (2.4) has a $2 n \times n$ matrix-valued solution $\left(S^{T}, C^{T}\right)^{T}(t)$ satisfying (2.6) or (2.7) for all $t \in \mathcal{I}$, then (2.4) is a trigonometric system. Moreover, this solution $\left(S^{T}, C^{T}\right)^{T}(t)$ is a conjoined basis of system (2.4).

Proof: By Lemma 2.5, it follows that (2.6) and (2.7) are equivalent. So it suffices to show that the results in this theorem hold under the assumption that system (2.4) has a $2 n \times n$ matrix-valued solution satisfying (2.7).

Suppose that $\left(S^{T}, C^{T}\right)^{T}(t)$ is a $2 n \times n$ matrix-valued solution of system (2.4) and satisfies (2.7). It follows from (2.7) that

$$
\left(\begin{array}{cc}
S & C \\
C & -S
\end{array}\right)(t+1)\left(\begin{array}{c}
S^{*} \\
C^{*}
\end{array}\right)(t+1)=\left(\begin{array}{c}
I_{n} \\
0
\end{array}\right)
$$

for all $t \in[a-1, \infty)$. Since $\left(S^{T}, C^{T}\right)^{T}(t)$ is a solution of system (2.4), we get from (2.10) that

$$
\left(\begin{array}{cc}
P S+Q C & -Q S+P C \\
-Q S+P C & -P S-Q C
\end{array}\right)(t)\left(\begin{array}{c}
S^{*} P^{*}+C^{*} Q^{*} \\
-S^{*} Q^{*}+C^{*} P^{*}
\end{array}\right)(t)=\left(\begin{array}{c}
I_{n} \\
0
\end{array}\right)
$$


for all $t \in \mathcal{I}$. So, for each $t \in \mathcal{I},(2.3)$ follows from (2.11) and (2.7). Further, by Lemma 2.5 , (2.2) holds for all $t \in \mathcal{I}$. Therefore, system (2.4) is a trigonometric system.

From (2.7) and the fact that $\operatorname{rank}\left(A A^{*}\right)=\operatorname{rank} A$ for any $n \times n$ matrix $A$, we have that $\operatorname{rank}(S, C)(t)=n$ for any $t \in \mathcal{I}$. Hence, $\left(S^{T}, C^{T}\right)^{T}(t)$ is a conjoined basis of system (2.4). The entire proof is complete.

\section{MAIN RESUlts}

In this section, we give the main results of this paper. First, we present two lemmas, which are useful in this section.

Lemma 3.1. Let $\left(X^{T}, U^{T}\right)^{T}(t)$ be any conjoined basis of system (1.2). Then there exists a $n \times n$ nonsingular matrix-valued function $H(t)$ satisfying

$$
\left(X^{*} X+U^{*} U\right)(t)=\left(H^{*} H\right)(t), \quad t \in \mathcal{I} .
$$

Further, it satisfies

$$
\left(X F X^{*}+U F U^{*}\right)(t)=I_{n},\left(U F X^{*}\right)(t)=\left(X F U^{*}\right)(t), \quad \forall t \in \mathcal{I},
$$

where $F(t):=\left(H^{*} H\right)^{-1}(t)$.

Proof: For simplicity, the variable $t$ is omitted in the proof. Denote $R:=\left(X^{*} X\right.$ $\left.+U^{*} U\right)$. Then it follows from (1) in Definition 2.1 that $\operatorname{rank} R=n$ and

$$
R=(X+i U)^{*}(X+i U) .
$$

This yields that $X+i U$ is nonsingular and $R>0$. So, there exists a unitary matrix-valued function $\hat{H}$ such that

$$
R=\widehat{H}^{*} \operatorname{diag}\left\{d_{1}, d_{2}, \ldots, d_{n}\right\} \widehat{H},
$$

where $d_{i}>0, i=1,2, \ldots, n$, are exactly eigenvalues of $R$. Set

$$
H:=\operatorname{diag}\left\{d_{1}^{1 / 2}, \dot{d}_{2}^{1 / 2}, \ldots, d_{n}^{1 / 2}\right\} \widehat{H} .
$$

It can be easily proved that (3.1) holds. Further, set $P=X H^{-1}$ and $Q=U H^{-1}$. By using the fact that $X^{*} U=U^{*} X$ and from (3.1), (2.2) follows. Hence, (2.3) holds by Lemma 2.5 and consequently, (3.2) holds. The proof is complete.

LEMma 3.2. Assume that $\left(X^{T}, U^{T}\right)^{T}(t)$ is the conjoined basis of system (1.2) and $\left(S^{T}, C^{T}\right)^{T}(t)$ is the conjoined basis of some trigonometric system (2.4) both with the initial condition (2.5). Let $H(t)$ be a $n \times n$ nonsingular matrix-valued function satisfying (3.1). If the following relations hold:

$$
X(t)=S^{*}(t) H(t), \quad U(t)=C^{*}(t) H(t),
$$


then the coefficient matrices $P$ and $Q$ in system (2.4) can be expressed as

$$
\begin{aligned}
& P(t)=H^{*-1}(t+1)\left(\begin{array}{l}
X \\
U
\end{array}\right) *(t) \Phi^{*}(t)\left(\begin{array}{l}
X \\
U
\end{array}\right)(t) H^{-1}(t) \\
& Q(t)=H^{*-1}(t+1)\left(\begin{array}{l}
X \\
U
\end{array}\right)(t) \Phi^{*}(t) J\left(\begin{array}{l}
X \\
U
\end{array}\right)(t) H^{-1}(t)
\end{aligned}
$$

ProOF: Since $\left(X^{T}, U^{T}\right)^{T}(t)$ is a solution of system (1.2), we have

$$
\left(\begin{array}{l}
X \\
U
\end{array}\right)^{*}(t+1)=\left(\begin{array}{l}
X \\
U
\end{array}\right)^{*}(t) \Phi^{*}(t)
$$

In addition, it follows from (3.3) that

$$
\left(\begin{array}{l}
X \\
U
\end{array}\right)^{*}(t+1)=H^{*}(t+1)(S, C)(t+1),
$$

which, together with (3.6), implies that

$$
(S, C)(t+1)=H^{*-1}(t+1)\left(\begin{array}{l}
X \\
U
\end{array}\right)^{*}(t) \Phi^{*}(t) .
$$

Multiplying (3.7) from the right by $\left(S^{* T}, C^{* T}\right)^{T}(t)$ and from (2.8), we have

$$
P(t)=H^{*-1}(t+1)\left(\begin{array}{l}
X \\
U
\end{array}\right)^{*}(t) \Phi^{*}(t)\left(\begin{array}{l}
S^{*} \\
C^{*}
\end{array}\right)(t),
$$

which, together with (3.3), yields that (3.4) holds. On the other hand, multiplying (3.7) from the right by $\left(C^{* T},-S^{* T}\right)^{T}(t)$ and from (2.9), we get

$$
Q(t)=H^{*-1}(t+1)\left(\begin{array}{l}
X \\
U
\end{array}\right)^{*}(t) \Phi^{*}(t)\left(\begin{array}{c}
C^{*} \\
-S^{*}
\end{array}\right)(t)
$$

Since it follows from (3.3) that

$$
J\left(\begin{array}{l}
X \\
U
\end{array}\right)(t) H^{-1}(t)=\left(\begin{array}{c}
C^{*} \\
-S^{*}
\end{array}\right)(t),
$$

(3.5) is derived from (3.8). This completes the proof.

REMARK 3.1. Lemma 3.2 is the discrete analog of [17, Lemma 2.3.1].

Lemma 3.2 only presents a relation between some special conjoined basis of system (1.2) and some special conjoined basis of a certain complex trigonometric system (2.4). However, we are enlightened by this result. Now, we can formulate one of the main results in the present paper-the Prüfer transformations for systems (1.1) and (1.2). 
THEOREM 3.1. For any conjoined basis $\left(X^{T}, U^{T}\right)^{T}(t)$ of system (1.1), or system (1.2), there exists a $n \times n$ nonsingular matrix-valued function $H(t)$ and $n \times n$ matrixvalued functions $S(t)$ and $C(t)$ such that (3.3) holds, where $\left(S^{T}, C^{T}\right)^{T}(t)$ is a conjoined basis of the complex trigonometric system (2.4) with $P(t)$ and $Q(t)$ being determined by (3.4) and (3.5), respectively, and $H(t)$ satisfies

$$
H(t+1)=(S, C)(t+1) \Phi(t)\left(\begin{array}{l}
S^{*} \\
C^{*}
\end{array}\right)(t) H(t), t \in \mathcal{I} .
$$

Proof: Based on the discussion in the first part of Section 2, it suffices to show that all the results hold for system (1.2).

Suppose that $\left(X^{T}, U^{T}\right)^{T}(t)$ is a conjoined basis of system (1.2). By Lemma 3.1, there exists a $n \times n$ nonsingular matrix-valued function $H(t)$ such that (3.1) and (3.2) hold. Set

$$
S(t):=\left(H^{*-1} X^{*}\right)(t), \quad C(t):=\left(H^{*-1} U^{*}\right)(t), \quad t \in \mathcal{I} .
$$

From (3.1) and by the fact that $\left(X^{*} U\right)(t)=\left(U^{*} X\right)(t)$ for all $t \in \mathcal{I}$, it follows that $\left(S^{T}, C^{T}\right)^{T}(t)$ satisfies (2.7) and consequently, it also satisfies (2.6) by Lemma 2.5. Therefore, from Theorem 2.1, if $\left(S^{T}, C^{T}\right)^{T}(t)$ is a matrix-valued solution of system (2.4) with $P(t)$ and $Q(t)$ being determined by (3.4) and (3.5), then (2.4) is a trigonometric system and $\left(S^{T}, C^{T}\right)^{T}(t)$ is a conjoined basis of (2.4) by using (3.1). So, it suffices to show that $\left(S^{T}, C^{T}\right)^{T}(t)$ is a matrix-valued solution of system (2.4).

In fact, from (3.4), (3.5), and (3.10), we have

$$
\begin{aligned}
\delta: & =P(t) S(t)+Q(t) C(t) \\
& =H^{*-1}(t+1)\left(\begin{array}{l}
X \\
U
\end{array}\right)^{*}(t) \Phi^{*}(t) \times\left[\left(\begin{array}{l}
X \\
U
\end{array}\right)(t) F(t) X^{*}(t)+J\left(\begin{array}{l}
X \\
U
\end{array}\right)(t) F(t) U^{*}(t)\right]
\end{aligned}
$$

where $F(t)$ is the same as in Lemma 3.1. Further, it follows from (3.2) that

$$
\begin{aligned}
\delta & =H^{*-1}(t+1)\left(\begin{array}{l}
X \\
U
\end{array}\right)^{*}(t) \Phi^{*}(t)\left(\begin{array}{l}
X F X^{*}+U F U^{*} \\
U F X^{*}-X F U^{*}
\end{array}\right)(t) \\
& =H^{*-1}(t+1)\left(\begin{array}{l}
X \\
U
\end{array}\right)^{*}(t) \Phi^{*}(t)\left(\begin{array}{c}
I_{n} \\
0
\end{array}\right) \\
& =H^{*-1}(t+1) X^{*}(t+1)=S(t+1),
\end{aligned}
$$

which implies that

$$
S(t+1)=P(t) S(t)+Q(t) C(t), t \in \mathcal{I} .
$$

Similarly, one can show that

$$
C(t+1)=-Q(t) S(t)+P(t) C(t), t \in \mathcal{I} .
$$


Hence, $\left(S^{T}, C^{T}\right)^{T}(t)$ is a matrix-valued solution of system (2.4) from (3.11) and (3.12).

Finally, it is to show that (3.9) holds. It follows from (3.10) that

$$
\left(\begin{array}{l}
S^{*} \\
C^{*}
\end{array}\right)(t+1) H(t+1)=\left(\begin{array}{l}
X \\
U
\end{array}\right)(t+1)=\Phi(t)\left(\begin{array}{l}
S^{*} \\
C^{*}
\end{array}\right)(t) H(t) .
$$

By multiplying (3.13) from the left by $(S, C)(t+1)$ and from (2.7), (3.9) is derived. The entire proof is complete.

REMARK 3.2. Theorem 3.1 extends [7, Theorem 2.3] for real symplectic systems to complex ones.

The final theorem presents discrete trigonometric transformations for systems (1.1) and (1.2), respectively, which are useful in the study of oscillation problems for complex discrete linear Hamiltonian systems and complex discrete linear symplectic systems.

THEOREM 3.2. For any pair of special normalised conjoined bases $\left(X_{1}^{T}, U_{1}^{T}\right)^{T}(t)$ and $\left(X_{2}^{T}, U_{2}^{T}\right)^{T}(t)$ of system (1.1) or system (1.2), there exist $n \times n$ matrix-valued functions $H(t)$ and $N(t)$ such that for each $t \in \mathcal{I}, H(t)$ is nonsingular, $H^{*}(t) N(t)$ is Hermitian, and the first components of $\left(X_{1}^{T}, U_{1}^{T}\right)^{T}(t)$ and $\left(X_{2}^{T}, U_{2}^{T}\right)^{T}(t)$ can be expressed as

$$
X_{1}(t)=H(t) S(t), \quad X_{2}(t)=H(t) C(t),
$$

where $\left(S^{T}, C^{T}\right)^{T}(t)$ is a conjoined basis of a trigonometric system (2.4) with the initial condition (2.5), while the coefficient matrices $P(t)$ and $Q(t)$ in system (2.4) can be expressed as

$$
\begin{aligned}
& P(t)=H^{-1}(t+1) E(t)[H(t)+B(t) N(t)] \\
& Q(t)=H^{-1}(t+1) E(t) B(t) H^{*-1}(t)
\end{aligned}
$$

for system (1.1) and

$$
\begin{aligned}
& P(t)=H^{-1}(t+1)\left[A_{11}(t) H(t)+A_{12}(t) N(t)\right], \\
& Q(t)=H^{-1}(t+1) A_{12}(t) H^{*-1}(t)
\end{aligned}
$$

for system (1.2), where $A_{11}(t)$ and $A_{12}(t)$ are the same as those in (2.1).

Proof: Based on the discussion in the first part of Section 2, it suffices to show that all the results hold for system (1.2). For simplicity, the variable $t$ is often omitted in the following discussion.

Suppose that $\left(X_{1}^{T}, U_{1}^{T}\right)^{T}(t)$ and $\left(X_{2}^{T}, U_{2}^{T}\right)^{T}(t)$ are any pair of special normalised conjoined bases of system (1.2). By Lemma 2.2, $\operatorname{rank}\left(X_{1}, X_{2}\right)=n$. Hence, $\operatorname{rank}\left(X_{1} X_{1}^{*}\right.$ $\left.+X_{2} X_{2}^{*}\right)=n$. It follows from Lemma 2.4 that

$$
X_{1} X_{1}^{*}+X_{2} X_{2}^{*}=\left(X_{1}+i X_{2}\right)\left(X_{1}+i X_{2}\right)^{*}
$$


which implies that $X_{1}+i X_{2}$ is nonsingular and $X_{1} X_{1}^{*}+X_{2} X_{2}^{*}>0$. So, there exists a nonsingular $n \times n$ matrix-valued function $H(t)$ such that

$$
X_{1} X_{1}^{*}+X_{2} X_{2}^{*}=H H^{*}
$$

Set

$$
S(t):=\left(H^{-1} X_{1}\right)(t), \quad C(t):=\left(H^{-1} X_{2}\right)(t),
$$

and

$$
N(t):=\left(U_{1} X_{1}^{*}+U_{2} X_{2}^{*}\right)(t) H^{*-1}(t) .
$$

It can be proved by Lemmas 2.1 and 2.4 and from (3.21) that

$$
H H^{*} N H^{*}-H N^{*} H H^{*}=0 .
$$

Hence,

$$
H^{*} N-N^{*} H=H^{-1}\left(H H^{*} N H^{*}-H N^{*} H H^{*}\right) H^{*-1}=0,
$$

that is, $H^{*}(t) N(t)$ is Hermitian and consequently,

$$
N H^{-1}=\left(N H^{-1}\right)^{*} .
$$

For convenience, denote $T(t):=\left(H H^{*}\right)^{-1}(t)$. Clearly, $T(t)=T^{*}(t)$ from (3.19). It follows from (3.21) and (3.22) that

$$
T X_{1}+N H^{-1} X_{2}=T\left(X_{1}+X_{1} U_{1}^{*} X_{2}+X_{2} U_{2}^{*} X_{2}\right) .
$$

Further, by Lemma 2.4 and from (3.19), we get

$$
\begin{aligned}
T X_{1}+N H^{-1} X_{2} & =T\left[X_{1}+X_{1}\left(X_{1}^{*} U_{2}-I_{n}\right)+X_{2} X_{2}^{*} U_{2}\right] \\
& =T T^{-1} U_{2}=U_{2} .
\end{aligned}
$$

Similarly, one can show that

$$
T X_{2}+N H^{-1} X_{1}=U_{1} .
$$

In addition, by using (3.20), (3.17), and (3.18),

$$
P(t) S(t)+Q(t) C(t)=H^{-1}(t+1) A_{11}(t) X_{1}(t)+H^{-1}(t+1) A_{12}(t)\left[N H^{-1} X_{1}+T X_{2}\right](t),
$$

which, together with (3.24), yields that

$$
P(t) S(t)+Q(t) C(t)=H^{-1}(t+1)\left(A_{11} X_{1}+A_{12} U_{1}\right)(t) .
$$


Since $\left(X_{1}^{T}, U_{1}^{T}\right)^{T}(t)$ is a solution of system (1.2), $X_{1}(t+1)=A_{11}(t) X_{1}(t)+A_{12}(t) U_{1}(t)$. So, we get from (3.25) and (3.20), that

$$
S(t+1)=P(t) S(t)+Q(t) C(t) .
$$

Similarly, using (3.23), one can get that

$$
C(t+1)=-Q(t) S(t)+P(t) C(t) .
$$

Hence, from (3.26) and (3.27), $\left(S^{T}, C^{T}\right)^{T}(t)$ is a $2 n \times n$ matrix-valued solution of system (2.4) with the coefficient matrices $P(t)$ and $Q(t)$ being determined by (3.17) and (3.18). Further, from (3.19) and (3.20) and by Lemma 2.4, it can be easily proved that $S(t)$ and $C(t)$ satisfy (2.7). Therefore, system (2.4) is a trigonometric system and $\left(S^{T}, C^{T}\right)^{T}(t)$ is a conjoined basis of system (2.4) by Theorem 2.1. This completes the proof.

REMARK 3.3. We now give an equivalent form to the trigonometric transformation (3.14). From (3.20), (3.22), and (3.24), we have

$$
\begin{aligned}
C & =H^{-1} X_{2} \\
& =H^{-1} X_{2}+N^{*} X_{1}-N^{*} X_{1} \\
& =H^{*} H^{*-1} H^{-1} X_{2}+H^{*} H^{*-1} N^{*} X_{1}-N^{*} X_{1} \\
& =-N^{*} X_{1}+H^{*}\left(T X_{2}+N H^{-1} X_{1}\right) \\
& =-N^{*} X_{1}+H^{*} U_{1} .
\end{aligned}
$$

So (3.14) can be written as

$$
\left(\begin{array}{l}
S \\
C
\end{array}\right)=\left(\begin{array}{cc}
H^{-1} & 0 \\
-N^{*} & H^{*}
\end{array}\right)\left(\begin{array}{l}
X_{1} \\
U_{1}
\end{array}\right) .
$$

Using a similar method to that used in [5, Lemma 7], one can show that the transformation (3.28) preserves oscillation behaviours of solutions of system (1.2). Hence, the transformation (3.14) preserves oscillation behaviours of solutions of systems (1.1) and (1.2).

REMARK 3.4. Theorem 3.2 extends [6, Theorem 3.1] for real symplectic systems to complex ones.

REMARK 3.5. The trigonometric system (2.4) can be turned into another trigonometric system with $Q$ replaced by a Hermitian and positive semi-definite matrix $\widetilde{Q}$ by a similar way to that used in [6, Remark 3.2].

\section{REFERENCES}

[1] R. Agarwal, C. Ahlbrandt, M. Bohner and A. Peterson, 'A survey paper on discrete linear Hamiltonian systems', Dynamic Systems Appl. 8 (1999), 307-333. 
[2] C.D. Ahlbrandt and A.C. Peterson, Discrete Hamiltonian systems: Difference equations, continued fractions, and Riccati equations (Kluwer Academic Publishers, Boston, 1996).

[3] D. Anderson, 'Discrete trigonometric matrix functions,', Panamer. Math. J. 7 (1997), 39-45.

[4] J.H. Barrett, 'A Prüfer transformation for matrix differential equations', Proc. Amer. Math. Soc. 8 (1957), 510-518.

[5] M. Bohner and O. Došlý, 'Disconjugacy and transformations for symplectic systems', Rocky Mountain J. Math. 27 (1997), 707-743.

[6] M. Bohner and O. Došlý, 'Trigonometric transformations of symplectic difference systems', J. Differential Equations 163 (2000), 113-129.

[7] M. Bohner and O. Došlý, 'Trigonometric systems in oscillation theory of difference equations', in Proc. Dynamic Systems and Applications 3 (Dynamic, Atlanta, GA, 2001), pp. 99-104.

[8] M. Bohner and O. Došlý, 'The discrete Prüfer transformation', Proc. Amer. Math. Soc. 129 (2001), 2715-2725.

[9] O. Došlý, 'On transformations of self-adjoint linear differential systems and their reciprocals', Ann. Polon. Math. 50 (1990), 223-234.

[10] W.G. Kelley and A.C. Peterson, Difference equations: an introduction with applications (Academic Press, San Diego, 1991).

[11] W. Kratz, Quadratic functionals in variational analysis and control theory (Akademie Verlag, Berlin, 1995).

[12] I. Rachunkova and C.C. Tisdell, 'Existence of non-spurious solutions to discrete boundary value problems', Aust. J. Math. Anal. Appl. 3 (2006), 1-9.

[13] W.T. Reid, 'A Prüfer transformation for differential systems', Pacific J. Math. 8 (1958), 575-584..

[14] Y. Shi, 'Weyl-Titchmarsh theory for a class of discrete linear Hamiltonian systems', Linear Algebra Appl. 416 (2006), 452-519.

[15] Y. Shi, Spectral theory of discrete Hamiltonian systems and stable initial manifolds for singularly perturbed systems, (Ph.D. thesis) (Shandong University, 2000).

[16] C.C. Tisdell, 'On first-order discrete bounary value problems', J. Difference Equ. Appl. 12 (2006), 1213-1223..

[17] Z. Zheng, The GKN theory, spectrum, and oscillation for linear Hamiltonian systems, (Ph.D. thesis) (Shandong University, 2003).

School of Statistics and Mathematics Shandong Economic University

Jinan

Shandong 250014

Peoples Republic of China

e-mail: yiwang8080@126.com
School of Mathematics and Systems Science Shandong University

Jinan

Shandong 250100

Peoples Republic of China

e-mail: ymshi@sdu.edu.cn renguojing@hotmail.com 